\title{
Vortices in thin ferromagnetic films and the skyrmion number
}

\author{
O. A. Tretiakov and O. Tchernyshyov \\ Department of Physics and Astronomy, The Johns Hopkins University, Baltimore, Maryland 21218
}

(Dated: January 23, 2007)

\begin{abstract}
We point out that a peculiar annihilation of a vortex-antovortex pair observed numerically by Hertel and Schneider [Phys. Rev. Lett. 97, 177202 (2006)] represents the formation and a subsequent decay of a skyrmion.

PACS numbers: 75.40.Gb, 75.40.Mg, 75.75.+a
\end{abstract}

Creation, annihilation, and fusion of topological solitons is constrained by conservation of related topological charges. For example, in a planar (XY) ferromagnet the destruction of a vortex always proceeds through its annihilation with an antivortex, a process that conserves the $\mathrm{O}(2)$ winding number. In this note we highlight the importance of another topological charge for vortex defects associated with the three-dimensional nature of the spin. Even in magnets with an easy-plane anisotropy the magnetization can and does point out of the plane at the core of a vortex. ${ }^{1}$ Even though the core region is exceedingly small, the direction of its out-of-plane magnetization $p=\operatorname{sgn} M_{z}(0)$, henceforth referred to as polarization, is an important parameter. For instance, the gyrotropic force acting on a moving vortex depends on the polar-

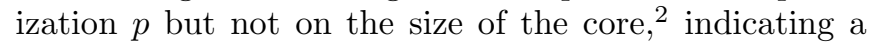
topological nature of the effect.

Recently Hertel and Schneider ${ }^{3}$ performed numerical simulations of the vortex-antivortex pair annihilation in a thin magnetic film. They noted drastically different outcomes for pairs with parallel and antiparallel core magnetizations. In the former case the two defects dissipated quietly, while in the latter the annihilation was accompanied by a violent burst of spin waves. Below we show that the difference is due to the conservation of another topological charge, the skyrmion number. Hertel and Schneider observed the formation and decay of a skyrmion.

In a thin film with no intrinsic anisotropy the magnetization is forced to stay mainly in the plane of the film by dipolar interactions. Therefore topological defects in the bulk of the film are characterized by an $\mathrm{O}(2)$ winding number, $n=+1$ for vortices and $n=-1$ for antivortices. At the core of these defects, the magnetization points out of the plane 1 As a result, there is a second topological invariant characterizing them, the skyrmion number

$$
q=\int \frac{d^{2} r}{8 \pi} \epsilon_{i j} \epsilon_{\alpha \beta \gamma} n_{\alpha} \partial_{i} n_{\beta} \partial_{j} n_{\gamma}=-\int \frac{d^{2} r}{4 \pi} \frac{\partial(\cos \theta, \phi)}{\partial(x, y)}
$$

where $\hat{\mathbf{n}}(\mathbf{r})$ is the unit vector parallel to the local magnetization $\mathbf{M}(\mathbf{r})$. Skyrmions were introduced in the context of the two-dimensional Heisenberg model by Belavin and

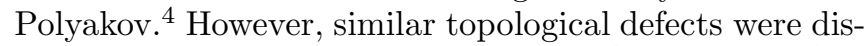

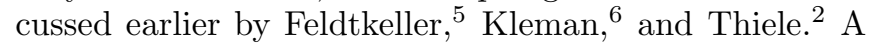
vortex with a winding number $n$ and core polarization $p$ has a half-integer skyrmion charge ${ }^{\frac{7}{}} q=n p / 2$. A vortexantivortex pair with parallel polarizations $p$ have oppo- site skyrmion numbers adding to zero and thus belongs to the same topological sector as uniform ground states. From the topological perspective, such a texture can be deformed continuously into a ground state and apparently this is exactly what happens: the energy decreases continuously until it reaches the ground-state value.

In contrast, a vortex and an antivortex with antiparallel core polarizations have equal skyrmion numbers adding to a total of +1 or -1 . This texture belongs to a nontrivial topological sector and thus cannot be deformed continuously into a ground state (with zero skyrmion number). Eventually the skyrmion decays into spin waves. A change in the topological sector requires the injection of a magnetic monopole,,$\frac{7}{-}$ also known as the Bloch point. 8 This process is strictly forbidden in continuum theories with a fixed length of magnetization but is allowed in lattice models .9 When the radius of a skyrmion shrinks to the lattice scale, the skyrmion can decay into spin waves. While details of this process depend on high-energy physics (lattice scale or the cost of locally suppressing magnetization length), the energy of spin waves released in the skyrmion decay can be readily estimated.

In the continuum approximation with exchange interactions only the energy is $E=A t \int d^{2} r|\nabla \hat{\mathbf{n}}|^{2}$, where $A$ is the exchange constant and $t$ is the film thickness. The local minima of energy in the topological sectors with $q= \pm 1$ are skyrmion textures with energy $\underline{4} 8 \pi A t$. Since the dipolar energy $\int d^{3} r \mu_{0}|\mathbf{H}(\mathbf{r})|^{2} / 2$ is positive definite, the exchange part provides a lower bound on the energy of a vortex-antivortex pair with opposite polarizations. Furthermore, the dipolar energy can be neglected when the skyrmion shrinks to the scale of the exchange length $\lambda=\sqrt{2 A / \mu_{0} M^{2}}$ (a few nanometers in permalloy).

For the values of exchange constant $A=1.3 \times$ $10^{-11} \mathrm{~J} / \mathrm{m}$ and film thickness $t=10 \mathrm{~nm}$ used in Ref. 3 we find $E_{\text {skyrm }} \approx 3.3 \times 10^{-18} \mathrm{~J}$. This matches well the energy of the vortex-antivortex pair just before the explosion $E_{\text {pair }} \approx 3.1 \times 10^{-18} \mathrm{~J}$ (measured from the ground state), see Fig. 4 in Ref. 3 .

Note added in proof: We have recently become aware of two more papers to which the subject of skyrmionmediated annihilation is relevant 10,11

The authors acknowledge support from the NSF Grant No. DMR-0520491 and from the Theoretical Interdisciplinary Physics and Astrophysics Center at JHU. 
1 T. Shinjo, T. Okuno, R. Hassdorf, K. Shigeto, and T. Ono, Science 289, 930 (2000).

2 A. A. Thiele, Phys. Rev. Lett. 30, 230 (1973); J. Appl. Phys. 45, 377 (1974).

${ }^{3}$ R. Hertel and C. M. Schneider, Phys. Rev. Lett. 97, 177202 (2006).

4 A. A. Belavin and A. M. Polyakov, Pis'ma ZhETF 22, 503 (1975) [JETP Lett. 22, 245 (1975)].

5 E. Feldtkeller, Z. Angew. Phys. 19, 530 (1965).

6 M. Kleman, Phil. Mag. 27, 1057 (1973).

7 T. Senthil, A. Vishwanath, L. Balents, S. Sachdev, and M. P. A. Fisher, Science 303, 1490 (2004).
8 A. Thiaville, J. M. García, R. Dittrich, J. Miltat, and T. Schrefl, Phys. Rev. B 67, 094410 (2003).

9 Ar. Abanov and V. L. Pokrovsky, Phys. Rev. B 58, R8889 (1998).

10 K.-S. Lee, S. Choi, and S.-K. Kim, Appl. Phys. Lett. 87, 192502 (2005).

11 B. Van Waeyenberge, A. Puzic, H. Stoll, K. W. Chou, T. Tyliszczak, R. Hertel, M. Fähnle, H. Brückl, K. Rott, G. Reiss, I. Neudecker, D. Weiss, C. H. Back, and G. Schütz, Nature (London) 444, 461 (2006). 